\title{
Parametric Description of Boiling and Cavitation
}

\author{
Liudmila V. Kulagina* \\ Siberian Federal University \\ 79 Svobodny, Krasnoyarsk, 660041, Russia
}

Received 19.01.2018, received in revised form 07.03.2018, accepted 06.05.2018

\begin{abstract}
Analysis of dimensions, physical examination of cavitation and boiling allowed revealing independent dimensionless thermodynamic parameters needed for describing these phenomena. In the most common case, only two thermodynamic parameters should be added to the previously introduced mechanical parameters.
\end{abstract}

Keywords: cavitation, boiling, independent dimensionless thermodynamic parameters.

Citation: Kulagina L.V. Parametric description of boiling and cavitation, J. Sib. Fed. Univ. Eng. technol., 2018, 11(5), 578-583. DOI: $10.17516 / 1999-494 X-0056$

\section{Параметрическое описание явлений}

\section{кипения и кавитации}

Л.В. Кулагина

Сибирский федеральный университет Россия, 660041, Красноярск, пр. Свободный, 79

Анализ размерностей, физическое исследование кавитации и кипения позволили установить независимые безразмерные термодинамические параметры, необходимые для описания указанных явлений. В наиболее распространенном случае к введенным ранее механическим параметрам следует добавить только два термодинамических параметра.

Ключевые слова: кавитация, кипение, независимые безразмерные термодинамические параметры.

\section{Введение}

В ходе изучения динамики образования и роста газовых пузырьков при кавитации или кипении обнаружено, что термодинамические эффекты способны играть при этом важную или даже преобладающую роль [1, 2]. Stepanoff A.J., Stai H.A. Salemann V., Spraker W.A. и другие исследователи [3-5] объединили эти эффекты, представив их единым безразмерным параметром,

C) Siberian Federal University. All rights reserved

* Corresponding author E-mail address: klvation@gmail.com 
обычно обозначаемым буквой $B$. Однако есть вероятность возможности одновременного влияния на рассматриваемые процессы двух параметров, которые, по аналогии, можно обозначить как $B^{\prime}$ и $B^{\prime \prime}$.

Настоящее исследование имеет целью показать, что в обычных случаях двух параметров достаточно для описания поведения сферической полости малых размеров в процессе ее роста при кипении или кавитации. Эти параметры должны быть добавлены к рассмотренным ранее параметрам, перечень которых определяется характером изучаемой проблемы. Они имеют отношение как к изучению зарождения кавитации, так и к проблеме эрозии, предположительно возникающей при разрыве полости малых размеров $[1,6]$. Речь здесь не идет о массопередаче за счет диффузии.

\section{Физические параметры}

К достаточно длинному перечню параметров, рассматриваемых при изучении динамики изменения объема сферического пузырька, необходимо добавить следующие термодинамические параметры [7]:

- скрытая теплота испарения (или конденсации) $L$;

- угол наклона кривой насыщения $p^{\prime}=\mathrm{d} p_{v} / \mathrm{d} T$;

- плотность пара $\rho_{\mathrm{v}}$;

- удельная теплоемкость жидкости $C_{i}$;

- удельные теплоемкости пара при постоянном объеме $C_{v v}$ и постоянном давлении $C_{v p}$;

- теплопроводность жидкости $\lambda_{i}$;

- теплопроводность пара $\lambda_{v}$;

- термодинамическая температура или разность температур.

Исключены случаи, связанные с наличием естественной конвекции и диффузии. Эта совокупность, включающая девять термодинамических параметров, сложна, но в ряде практически наиболее важных случаев некоторыми из них можно пренебречь. Это прежде всего в тех случаях, когда температура не слишком близка к критическому значению, энергообмен между двумя фазами зависит в основном от теплоты испарения $L$, а вклад теплоемкости пара пренебрежимо мал. С другой стороны, М.С. Плессет показал [8], что в большинстве случаев температура газовой фазы постоянна, так что теплопередача здесь пренебрежимо мала и теплопроводность пара $\lambda_{v}$ не оказывает влияния на рассматриваемое явление (случай изотермического течения).

Следовательно, в подобных случаях параметры $C_{v v}, C_{v p}$ и $\lambda_{v}$ можно исключить не потому, что в исследуемой области условий они остаются постоянными (это исказило бы последующий анализ), а в связи с тем, что представляемые ими физические явления не играют значительной роли.

\section{Анализ параметров}

Представленный ниже анализ - не чистый анализ размерностей, который не дал бы возможности в достаточной степени сократить число независимых параметров. Он включает также физический анализ явления, особенно в том виде, как оно представлено в публикациях Ж. Бонэна $[7,9,10]$. Как и анализ размерностей, данный анализ дифференциальный, 
т.е. позволяет не определять полностью систему переменных и безразмерных параметров (достаточно уточнить, что речь идет о сложной динамической системе с распределенными параметрами [11, 12]).

С учетом исключений, указанных в конце предыдущего раздела, начальная динамическая система может быть охарактеризована при помощи следующих шести физических величин: $L$, $C, k, T, \rho_{v}, \rho_{\mathrm{v}}$ '. Наряду с такими исключениями дополнительно введен только один независимый параметр - температура (действительно, теплота эквивалентна механической энергии).

Прежде чем применить к данной системе дифференциальную форму $\pi$-теоремы ВашиБакингема-Рябушинского $[13,14]$, которая в общем случае позволяет установить общую структуру зависимости, вытекающую только лишь из требования инвариантности физической зависимости при изменении масштабов единиц, даже если конкретный вид зависимости между исходными величинами неизвестен, можно высказать следующие соображения, каждое из которых позволяет исключить один из независимых параметров.

1. Наклон кривой насыщения $\rho_{\mathrm{v}}{ }^{\prime}$ не является независимой величиной, так как он связан с $L, T, \rho_{\mathrm{v},} \rho_{l}\left(\rho_{l}-\right.$ плотность жидкости, всегда присутствующей в «полной» динамической системе) уравнением Клайперона-Клаузиса:

$$
L=T \rho_{\mathrm{v}}{ }^{\prime}\left(1 / \rho_{\mathrm{v}}-1 / \rho_{l}\right)
$$

2. Как показал физический анализ $[7,9,10,15]$ явления, величина $L$ выступает не сама по себе, а в виде произведения на плотность пара $\rho_{\mathrm{v}}$.

3. Тот же физический анализ показал, что отношение плотностей двух фаз $\varepsilon=\rho_{\mathrm{v}} / \rho_{l}$ всегда находится в составе произведения. Даже если сохранить величину $\varepsilon$ в качестве безразмерного параметра анализа, ее влияние на явление будет заранее известно из других источников.

С учетом этих соображений применение $\pi$-теоремы приводит к определению двух новых безразмерных параметров, не зависимых один от другого и учитывающих все термодинамические переменные. Речь идет о введенных ранее параметрах $B$ ' и $B^{\prime \prime}$.

\section{Выбор безразмерных параметров}

Как показано выше, теоретически можно рассматривать группу из двух (или из трех, включая ع) независимых безразмерных параметров, учитывающих все термодинамические переменные. Разумеется, эти термодинамические параметры должны дополнять динамические переменные. Одни группы могут оказаться более полезными, чем другие, при анализе экспериментальных результатов.

Может быть целесообразным объединение в одну группу характеристик, относящихся только к жидкости. Это было сделано посредством введения понятий характеристического времени $\tau$ и характеристического давления $p_{c}$ после введения понятия двухфазного сопротивления $\psi$ :

$$
\begin{aligned}
& \psi=L \varepsilon \rho_{\mathrm{v}}{ }^{\prime}, \\
& \tau=\mathrm{K} / \psi=\mathrm{K} / L \varepsilon \rho_{\mathrm{v}}{ }^{\prime}, \\
& p_{c}=\psi / \mathrm{C}=L \varepsilon \rho_{\mathrm{v}}{ }^{\prime} / \mathrm{C} .
\end{aligned}
$$


Поскольку изучаемое давление может, например, быть следствием изменения давления $\Delta p$ на протяжении периода времени $t$, параметры $B$ ' и $B$ ” логично представить следующим образом:

$$
\begin{aligned}
& B^{\prime}=t / \tau, \\
& B^{\prime \prime}=\Delta p / p_{c} .
\end{aligned}
$$

Ранее показано [7], что изменения $\tau$ и $p_{c}$ весьма значительны и могут быть представлены, например, изменениями $\psi$. Кроме того, было признано целесообразным сохранить эти существенные изменения в одном параметре, например в $\tau$ или $\psi$, и учесть остальные термодинамические величины при помощи другого параметра, изменения которого ограничены. Физический анализ $[1,2,13]$ указывает также на влияние температуропроводности жидкости

$$
a_{l}=k_{l} / \rho_{l} C_{l}
$$

которая имеет размерность кинематической вязкости. Таким образом, в явлениях инициирования кавитации или кавитационной эрозии можно сохранить параметр $B^{\prime}$ и другой безразмерный параметр, построенный с использованием величины «а».

Если динамический анализ проблемы указывает на необходимость рассмотрения вязкости $v$, этот новый параметр, очевидно, принимает следующий вид:

$$
v / a=\operatorname{Pr},
$$

т.е. представляет собой число Прандтля для жидкости. Этот результат не вызывает удивления. Действительно, поскольку поток зависит от вязкостных сил, кавитация и кипение жидкости под действием тепловых явлений могут быть представлены только для жидкости, число Прандтля у которой близко к некоторому определенному значению. В частности, жидкий металл можно уподобить только другому жидкому металлу.

В некоторых случаях влияние вязкости является косвенным и, как показало предыдущее исследование, пренебрежимо малым. Если речь идет о потоке, можно сформировать с использованием величины «a» параметр, аналогичный числу Рейнольдса:

$$
\operatorname{Re}=x V / a .
$$

Этот параметр необходимо принимать во внимание наряду с действующими термодинамическими факторами.

С другой стороны, при изучении эрозии в вибрационной системе с периодом $t$ и амплитудой $x$ полезно дополнить параметр $t / \tau$ новым параметром

$$
x^{2} / a t \text {. }
$$

Из этого следует, что даже в тех случаях, когда не приходится прибегать к числу Прандтля, трудно заменить одну жидкость другой с существенно отличной температуропроводностью (не всегда допустимы значительные изменения значений $x$ и $t$ под влиянием динамических элементов, которые здесь не уточнены и рассмотрены в работе А. Тирувенгадама [6]). 
Некоторые исследователи, например В.В. Mikic, W.M. Rosenow, P. Griffith [16], использовали число Якоби, которое может быть представлено в форме

$$
\mathrm{J} a=C_{l} \Delta T / L \varepsilon,
$$

особенно полезной при изучении динамики кипения. Действительно, комплекс $L \varepsilon / C_{l}$ можно рассматривать как характеристическую температуру жидкости. Следовательно, число Якоби эквивалентно предложенному параметру $B$ ”. Однако при исследовании кавитации целесообразнее использовать отношение давлений.

\section{Увеличение числа параметров}

Если температура достаточно близка к критическому значению, вследствие чего начинает проявляться влияние теплоемкости пара, необходимо ввести два новых безразмерных параметра (отношения теплоемкостей): $C v p / C_{l}$ и, очевидно, $\gamma=C v p / C v v$. С другой стороны, при наличии влияния динамики теплообмена в паровой фазе (внутри пузырька) приходится рассматривать еще один безразмерный параметр, а именно отношение теплопроводностей двух фаз $-k_{v} / k_{l}$.

\section{Выводы}

В ходе настоящей работы удалось показать, что различные термодинамические величины, способные влиять на динамику кипения и кавитацию, можно в наиболее распространенных случаях свести к двум независимым безразмерным параметрам, по меньшей мере один из которых включает отношение плотностей двух фаз $\varepsilon$. Эти параметры необходимо добавить к механическим параметрам, относящимся к рассматриваемой задаче.

Безусловно, выбор этих параметров является относительно произвольным. Такой выбор может и должен быть сделан с учетом характера изучаемой задачи. В частности, для изучения кавитации больше подходит параметр, включающий разность давлений, а для изучения кипения - параметр, включающий разность температур.

В некоторых случаях, рассмотренных в ходе настоящего исследования, в частности при близости температуры к критическому значению, приходится дополнительно вводить другие параметры. Однако представляется вполне вероятным, что детальный анализ подобных случаев приведет к другим упрощениям.

Наконец, следует отметить, что возможное вмешательство естественной конвекции потребует, очевидно, введения соответствующего параметра, например числа Грасгофа.

Исследование выполнено при финансовой поддержке РФФИ и Правительства Красноярского края в рамках научных проектов №№ 17-48-240386, 18-48-242001 и 18-41-242004.

\section{Список литературы}

[1] Ивченко В.М., Кулагин В.А., Немчин А.Ф. Кавитационная технология; ред. акад. Г.В. Логвинович. Красноярск: Изд-во КГУ, 1990. 200 с. [Ivchenko V.M., Kulagin V.A., Nemchin A.F. Cavitation technology; Ed. acad. G.V. Logvinovich. Krasnoyarsk: Publishing House of KSU, 1990. 200 p. (in Russian)]. 
[2] Кнепп Р., Дейли Дж., Хэммит Ф. Кавитация. М.: Мир, 1974. 688 с. [Knapp R., Daily J., Hammit F. Cavitation. M.: Mir, 1974. 688 p. (in Russian)].

[3] Stepanoff A.J., Stai H.A. Thermodynamic Aspekts of Cavitation in Centrifugal Pumps, Trans. ASME, Series A, 1956, 78, November, 1691.

[4] Salemann V. Cavitation and N.P.S.H. Requirements of various Liquids, A.S.M.E. Journal of basic Engineering, Join, 1959, 167.

[5] Spraker W.A. The Effect of Fluid Properties on Cavitation in Centrifugal Pumps, A.S.M.E., Ser. E, 1965, 87, Juliet.

[6] Tiruvengadam A. Scaling, Laws for Erosion. Hydronautics Inc. Technical Report 233-15.

[7] Bonnin J. Notion de resistance diaphasique Application a la I cavitation cl a I'cbullition - E.d. Fi Bulletin tic la Direction des Eludes ct Recherchcs. Serie A. n. 1, 1972, 39-76.

[8] Plesset M.S. Bubble Dynamics. Cavitation in Real Fluids, Warren Michigan USA. 1962. Elsilver 1964.

[9] Bonnin J. Influence de la temperature sur le debut de cavitation dans l'eau, Societe Hydrotechniquc de France, XII journees de I'Hydrauliquc, Paris, join, 1972.

[10] Bonnin I. Thermodynamic Effect in Cavitation. Fluid Machinery, Group Conference “Cavitation", September, 1974, Heriot-Wait University - Edinburg.

[11] Демиденко Н.Д., Кулагин В.А., Шокин Ю.И., Ли Ф.-Ч. Теплообмен и суперкавитация. Новосибирск: Наука, 2015. 436 с. [Demidenko N.D., Kylagin V.A., Shokin U.I., Lee F.-C. Heat and Mass Transfer and Supercavitation. Novosibirsk: Nauka, 2015. 436 p. (in Russian)].

[12] Демиденко Н.Д., Кулагина Л.В. Моделирование и оптимизация технических систем с распределенными параметрами. Красноярск: ИПЦ КГТУ, 2006. 210 с. [Demidenko N.D., Kulagina L.V. Simulation and Optimization of Engineering Systems with Distributed Parameters. Krasnoyarsk, Publishing Center KSTU, 2006. 210 p. (in Russian)].

[13] Седов Л.И. Методы подобия и размерности в механике. М.: Наука, 1981. С. 31.448 с. [Sedov L.I. Similarity and dimension methods in mechanics. M.: Nauka, 1981. 448 p. (in Russian)].

[14] Бриджмен П. Анализ размерностей. Ижевск: РХД, 2001. 148 с. [Bridgman P. Analysis of dimensions. Izhevsk: RHD, 2001. 148 p. (in Russian)].

[15] Bonnin J. Theoretical and Experimental Investigations on Incipient Cavitation in Different Liquids, A.S.M.E. Winter Meeting. New York, November, 1972. Paper 72-WA/FE 31.

[16] Mikic B.B., Rosenow W.M., Griffith P. On Bubble Growth Rate, Int. Journal Heat Mass Transfer, 1970, 13, 657-666. 